\title{
Circular edge states in photonic crystals with a Dirac node
}

\author{
K. Ziegler \\ Institut für Physik, Universität Augsburg \\ D-86135 Augsburg, Germany
}

(Dated: September 11, 2018)

\begin{abstract}
Edge states are studied for the two-dimensional Dirac equation in a circular geometry. The properties of the two-component electromagnetic field are discussed in terms of the three-component polarization field, which can form a vortex structure near the Dirac node with a vorticity changing with the sign of the Dirac mass. The Berry curvature of the polarization field is related to the Berry curvature of the Dirac spinor state. This quantity is sensitive to a change of boundary conditions. In particular, it vanishes for a geometry with a single boundary but not for a geometry with two boundaries. This effect is robust against the creation of a step-like edge inside the sample.
\end{abstract}

\section{INTRODUCTION}

Recent progress in the design of photonic metamaterials has opened a new research area in which many ideas, originally developed for quantum field theories or for electronic systems, can be realized and tested with photons under much more general conditions than originally anticipated. A crucial advantage of photonics in comparison to electronics is that photons are not charged and do not interact directly with each other. Moreover, photons cover a wide range of length scales which are described by the same Maxwell theory. This provides the opportunity to design metamaterials from nano- to centimeter length scales for the same type of physics. For example, there have been experiments with visible light as well as with microwaves in the frequency regime down to $10 \mathrm{GHz}$. Thus, the characteristic lengths in the photonic experiments vary from $10^{-9} \mathrm{~m} \ldots 10^{-2} \mathrm{~m}$.

The analogy between electronic materials and photonic systems in dielectric media has lead to a number of interesting experiments. Starting from the popular case of graphene, where the underlying honeycomb lattice is formed by carbon atoms, exceptionally robust electronic transport properties have been observed [1]. This is caused by spectral degeneracies in the form of Dirac nodes, which were also studied recently in photonic crystals [2-11]. In analogy to the robust electronic transport, the photonic experiments indicate very robust photonic edge states, protected by topological invariants, such as the Chern number [2]. However, in the presence of time-reversal symmetry, as it exists in photonic lattices with a real dielectric constant, Dirac nodes appear only pairwise with opposite sign of the Dirac mass. This implies that the Chern number, which is an integral over the entire Brillouin zone, vanishes in this case. Breaking this symmetry, for instance through the Faraday effect, can result in a change of the Chern number [2]. Several procedures are discussed in the recent literature, how this can be achieved in photonic crystals and metamaterials [7-11]. A typical procedure for the creation of edge states in systems with Dirac nodes consists of three steps: (i) opening of a gap at the Dirac nodes, (ii) changing the Chern number by breaking the time-reversal symmetry and (iii) removing all Dirac nodes except for a single one. Although the last step is not necessary, it is possible [12-14] and simplifies the theoretical discussion.

The following study has been motivated by a series of experiments with edge states in photonic crystals near a slightly gapped Dirac node [7, 9, 11]. Depending on the sign of the gap parameter (or Dirac mass), there is a positive or a negative Chern number associated with this sign [2]. The Dirac mass may change in space such that positive and negative spatial regions can be distinguished. It is possible to design a photonic crystal with several regions of positive and negative Dirac mass, and edge states between these regions [11]. The aim of this work is to study the properties of edge states in different geometries, how they are affected by the geometry of the boundaries, and to propose a method how to characterize them through the polarization of the electromagnetic field.

In the following we distinguish two different types of edges, namely sample boundaries and edges created by a sign change of the Dirac mass. For clarity, we will use the name edge only for the latter but reserve boundaries for the sample geometry. In agreement with the experimental studies, we consider a two-dimensional photonic crystal with a single Dirac node [2-11] and assume a small gap. The twocomponent electromagnetic field in the $x-y$ plane is a solution of the two-dimensional (2D) Dirac equation and represents a Dirac spinor in a formal sense. The solutions of the corresponding Helmholtz equation are well known and can be found in text books (e.g., Ref. [15]). Although the purpose of this short note 
is to describe edge states in a photonic crystal near a Dirac node, the results should also be applicable to electronic quasiparticles with a cone-like spectrum. However, the effect of the Coulomb interaction must be included in the electronic case, which will not be considered here.

The Berry phase [16] or the Chern number, both obtained by integration, have been used to characterize the properties of the spinor eigenstate of the Hamiltonian as a function of the Dirac mass and the band index (upper or lower band) $[2,11,17]$. Here we employ an alternative approach with the polarization field. This is a three-component rather than a two-component vector. A connection between the polarization and the Berry curvature was discussed earlier for an optical fiber in Ref. [18].

\section{2D DIRAC EQUATION}

We consider a photonic crystal which is characterized by a periodic space-dependent dielectric tensor $\epsilon$. This implies that the spectrum of the Maxwellian has a band structure [19, 20]. As mentioned in the Introduction, it was shown that for a hexagonal arrangement of dielectric rods or cylinders there are two spectral nodes at $\omega=\omega_{D}$ in the band structure $[2,7]$. Near these spectral nodes the Maxwell equation is reduced for the TE (transverse electric) state to two 2D Dirac equations

$$
H_{D ; \pm} \mathbf{E}_{ \pm}=0, \quad H_{D ; \pm}=\left(\begin{array}{cc}
m & i \partial_{x} \pm \partial_{y} \\
i \partial_{x} \mp \partial_{y} & -m
\end{array}\right)
$$

$\partial_{x} \equiv \partial / \partial x, \ldots$. All lengths are measured in units of $c / \sqrt{\epsilon} \omega$, where $c$ is the speed of light, $\omega$ the frequency of the electromagnetic field and $\epsilon$ the dielectric constant. The degeneracy of the two Dirac equations with $H_{D ; \pm}$ can be lifted by introducing different masses $m_{ \pm}$, where one mass is much bigger than the other. For a proper frequency $\omega_{D}$ there is only one Dirac node (with massless or almost massless) Dirac particles $[2,7]$. The eigenvalue problem of the Dirac operator

$$
H_{D} \mathbf{E}=\left(\begin{array}{cc}
m & i \partial_{x}+\partial_{y} \\
i \partial_{x}-\partial_{y} & -m
\end{array}\right)\left(\begin{array}{c}
E_{\uparrow} \\
E_{\downarrow}
\end{array}\right)=E\left(\begin{array}{c}
E_{\uparrow} \\
E_{\downarrow}
\end{array}\right)
$$

on a square geometry is solved by

$$
\mathbf{E}=\left(\begin{array}{c}
E_{\uparrow} \\
E_{\downarrow}
\end{array}\right)=\frac{1}{\sqrt{2}}\left(\begin{array}{c}
\frac{k_{x}-i k_{y}}{-E+m} \\
1
\end{array}\right) e^{i k_{x} x+i k_{y} y}
$$

with the condition

$$
E^{2}=k_{x}^{2}+k_{y}^{2}+m^{2}
$$

for the wavevector $\mathbf{k}=\left(k_{x}, k_{y}\right)$, which is adapted to the boundary conditions. This result describes two-dimensional Dirac photons for energies $|E| \geq|m|$.

An electromagnetic field is characterized by four Stokes parameters [21, 22]. They can be expressed as quadratic forms of the electric field $\mathbf{E}$ with Pauli matrices $\sigma_{j}\left(j=0, x, y, z ; \sigma_{0}\right.$ is the $2 \times 2$ unit matrix): $I=\left(\mathbf{E} \cdot \sigma_{0} \mathbf{E}\right)$ is the intensity with the two-dimensional scalar product $(. \cdot$.$) and$

$$
Q=\left(\mathbf{E} \cdot \sigma_{z} \mathbf{E}\right), \quad U=\left(\mathbf{E} \cdot \sigma_{x} \mathbf{E}\right), \quad V=\left(\mathbf{E} \cdot \sigma_{y} \mathbf{E}\right)
$$

are the other Stokes parameters, which provide the polarization. It should be noticed that the relation $Q^{2}+U^{2}+V^{2}=I^{2}$ implies that the tip of the vector $(Q, U, V)$ describes a sphere of radius $I$ (Poincaré sphere). After normalizing the radius to 1 , the vector field

$$
\mathbf{n}=\frac{1}{I}\left(\begin{array}{l}
U \\
V \\
Q
\end{array}\right)
$$

can be used to characterize the polarization through a Berry curvature. This will be briefly discussed in Sect. IV. 


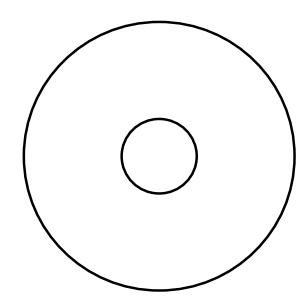

a

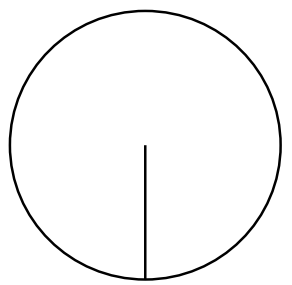

b

FIG. 1: Geometry of circular samples. Sample a has two circular boundaries, whereas sample b with the radial cut has a single boundary.

For the plane wave solution of Eq. (3) we get for the Stokes parameters in $\mathbf{k}$-space expressions which are uniform in $\mathbf{r}-$ space

$$
I=\frac{E}{E-m}, \quad\left(\begin{array}{l}
U \\
V
\end{array}\right)=-\frac{\mathbf{k}}{E-m}, \quad Q=\frac{m}{E-m}
$$

as the result of the translational invariance of the system and the square geometry. In the presence of an edge or in a circular geometry this translational invariance is broken. As a consequence, the Stokes parameters will become $\mathbf{r}$ dependent.

\section{A. Circular geometry}

First, we consider states outside the gap (i.e., for $E^{2}>m^{2}$ ) in a disc geometry. The Dirac eq. (2) reads in terms of polar coordinates $(x, y)=(r \cos \alpha, r \sin \alpha)$

$$
\left(\begin{array}{cc}
m & i e^{-i \alpha}\left(\partial_{r}-\frac{i}{r} \partial_{\alpha}\right) \\
i e^{i \alpha}\left(\partial_{r}+\frac{i}{r} \partial_{\alpha}\right) & -m
\end{array}\right)\left(\begin{array}{c}
E_{1} \\
E_{2}
\end{array}\right)=E\left(\begin{array}{c}
E_{1} \\
E_{2}
\end{array}\right)
$$

Assuming that the solution $E_{1}$ is rotational invariant at the boundary of the disc, this equation is solved by

$$
\left(\begin{array}{c}
E_{1} \\
E_{2}
\end{array}\right)=\left(\begin{array}{c}
a J_{0}(\bar{r})+b Y_{0}(\bar{r}) \\
i e^{i \alpha} \frac{\sqrt{E^{2}-m^{2}}}{E-m}\left[a J_{1}(\bar{r})+b Y_{1}(\bar{r})\right]
\end{array}\right)
$$

where $\bar{r}=\sqrt{E^{2}-m^{2}} r$, and $J_{n}, Y_{n}$ are Bessel functions. The coefficients $a$ and $b$ must be fixed by the specific boundary conditions of $\mathbf{E}$. Then the Stokes parameters of this solution are

$$
I=\left(a J_{0}+b Y_{1}\right)^{2}+\left(a J_{1}+b Y_{1}\right)^{2}, \quad\left(\begin{array}{l}
U \\
V
\end{array}\right)=2 \frac{\sqrt{E^{2}-m^{2}}}{r(E-m)}\left(a J_{0}+b Y_{1}\right)\left(a J_{1}+b Y_{1}\right)\left(\begin{array}{c}
-y \\
x
\end{array}\right)
$$

and

$$
Q=\left(a J_{0}+b Y_{1}\right)^{2}-\left(a J_{1}+b Y_{1}\right)^{2} .
$$

$I$ and $Q$ are rotational invariant (i.e., they do not depend on $\alpha$ ), and the other two Stokes parameter describe a circulating polarization in the $x-y$ plane around the center of the disc. Since the sign of the vorticity changes as a function of the radius because of changing signs of the Bessel functions, this structure does not represent a vortex.

The situation is different inside the gap (i.e., for $E^{2}<m^{2}$ ), where states decay exponentially. For example, we consider the zero energy state of the Dirac eq. (8) for the two geometries of Fig. 1. For Fig. 1a with two boundaries we assume $E_{1}$ is rotational invariant at both boundaries. Then the solution reads

$$
\left(\begin{array}{c}
E_{1} \\
E_{2}
\end{array}\right)=\left(\begin{array}{c}
K_{0}(\bar{r}) \\
-i e^{i \alpha} \operatorname{sgn} m K_{1}(\bar{r})
\end{array}\right)
$$



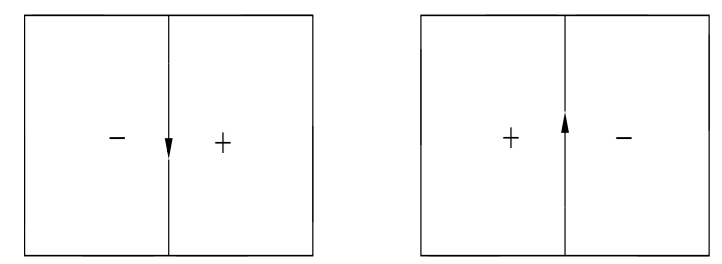

FIG. 2: A straight edge, where the arrows indicate the polarization. The \pm are the signs of the Dirac mass.

where $K_{n}$ are modified Bessel functions and $\bar{r}=|m| r$. The inner circle in Fig. 1a is necessary, since $K_{0}$, $K_{1}$ diverge for $r \sim 0$. In more physical terms, the inner boundary creates the edge state. The Stokes parameters read in this case

$$
I=K_{0}^{2}+K_{1}^{2}, \quad\left(\begin{array}{l}
U \\
V
\end{array}\right)=-2 \operatorname{sgn} m \frac{K_{0} K_{1}}{r}\left(\begin{array}{c}
-y \\
x
\end{array}\right), \quad Q=K_{0}^{2}-K_{1}^{2},
$$

where $U$ and $V$ represent a vortex solution in the plane with a vorticity proportional to sgnm, since $K_{0} K_{1}>0$.

For the simply connected geometry with a radial cut of Fig. 1b the Dirac eq. (8) is solved by

$$
\left(\begin{array}{c}
E_{1} \\
E_{2}
\end{array}\right)=\frac{1}{\sqrt{2}}\left(\begin{array}{c}
e^{-i \alpha / 2} \\
-i \operatorname{sgn} m e^{i \alpha / 2}
\end{array}\right) \frac{e^{-|m| r}}{\sqrt{r}}
$$

where the angular function $e^{ \pm i \alpha / 2}=\sqrt{x \pm i y} / \sqrt{r}$ has a branch cut. This must be associated with boundary conditions such that the phase of the electric field has a $\pi$ jump at the radial cut in Fig 1 b. The intensity and the other Stokes parameters are not affected by the branch cut:

$$
I=\frac{e^{-2|m| r}}{r}, \quad\left(\begin{array}{l}
U \\
V
\end{array}\right)=-\operatorname{sgn} m\left(\begin{array}{c}
-y \\
x
\end{array}\right) \frac{e^{-2|m| r}}{r^{2}}, \quad Q=0 .
$$

Again, $U, V$ represent a vortex in the plane with a vorticity proportional to the sign of the Dirac mass. Thus, the Stokes parameters agree qualitatively for the two circular geometries. However, the parameter $Q$ (the third component of the polarization, according to Eq. (5)) vanishes only for the simply connected geometry, whereas $Q<0$ for the geometry with two boundaries.

\section{EDGE STATES WITH 2D DIRAC PHOTONS}

\section{A. Straight edge}

Now we consider a space-dependent Dirac mass which forms an edge along the $y$-direction with $-m$ $(m)$ for $x<0(x \geq 0)$, as visualized in Fig. 2. This straight edge creates a zero energy state [23]

$$
\left(\begin{array}{l}
E_{1} \\
E_{2}
\end{array}\right)=\frac{1}{\sqrt{2}}\left(\begin{array}{c}
i \operatorname{sgn} m \\
1
\end{array}\right) e^{-|m x|}
$$

inside the gap of width $2|m|$. For this edge state we have $Q=0$, and the remaining Stokes parameters describe a global polarization in the $x-y$ plane:

$$
I=e^{-2|m x|}, \quad\left(\begin{array}{l}
U \\
V
\end{array}\right)=-\left(\begin{array}{c}
0 \\
\operatorname{sgn} m
\end{array}\right) e^{-2|m x|}, \quad Q=0 .
$$

Thus, the polarization is directed along the $y$-axis, where the sign of $m$ determines its orientation (cf. Fig. 2). 


\section{B. Circular edge for a geometry with two circular boundaries}

Now we return to the circular geometries of Fig. 1 and add an edge that forms a loop inside a sample. For simplicity, we assume a concentric circular edge of radius $r_{0}$, where the Dirac mass $m$ jumps from the inside $\left(m=m_{i}\right)$ to the outside region $\left(m=-m_{i}\right)$, as sketched by the dashed circles in Fig. 3 . Then the zero energy state of the Dirac eq. (8) is solved by the spinor $\mathbf{E}$ with components

$$
\left(\begin{array}{l}
E_{1} \\
E_{2}
\end{array}\right)=\left(\begin{array}{c}
a K_{0}(\bar{r})+b I_{0}(\bar{r}) \\
i e^{i \alpha} \operatorname{sgn} m_{i}\left[-a K_{1}(\bar{r})+b I_{1}(\bar{r})\right]
\end{array}\right) \quad\left(r \leq r_{0}\right)
$$

and

$$
\left(\begin{array}{l}
E_{1} \\
E_{2}
\end{array}\right)=\left(\begin{array}{c}
c K_{0}(\bar{r}) \\
i e^{i \alpha} \operatorname{sgn} m_{i} c K_{1}(\bar{r})
\end{array}\right) \quad\left(r>r_{0}\right) .
$$

$K_{n}$ and $I_{n}$ are modified Bessel functions, $\bar{r}=\left|m_{i}\right| r$. The coefficients

$$
a=\frac{I_{1}\left(\bar{r}_{0}\right) K_{0}\left(\bar{r}_{0}\right)-I_{0}\left(\bar{r}_{0}\right) K_{1}\left(\bar{r}_{0}\right)}{I_{1}\left(\bar{r}_{0}\right) K_{0}\left(\bar{r}_{0}\right)+I_{0}\left(\bar{r}_{0}\right) K_{1}\left(\bar{r}_{0}\right)}, \quad b=\frac{2 c}{I_{0}\left(\bar{r}_{0}\right) / K_{0}\left(\bar{r}_{0}\right)+I_{1}\left(\bar{r}_{0}\right) / K_{1}\left(\bar{r}_{0}\right)}
$$

secure a continuous behavior of the spinor at the edge $r=r_{0}$, and the coefficient $c$ must be fixed by the boundary value of $\mathbf{E}$ at the inner circular boundary.

From the solutions $(18,(19)$ we obtain the Stokes parameters as

$$
\begin{gathered}
I=\left\{\begin{array}{ll}
\left(a K_{0}+b I_{0}\right)^{2}+\left(-a K_{1}+b I_{1}\right)^{2} & \text { for } r \leq r_{0} \\
c^{2}\left(K_{0}^{2}+K_{1}^{2}\right) & \text { for } r>r_{0}
\end{array},\right. \\
Q=\left\{\begin{array}{ll}
\left(a K_{0}+b I_{0}\right)^{2}-\left(-a K_{1}+b I_{1}\right)^{2} & \text { for } r \leq r_{0} \\
c^{2}\left(K_{0}^{2}-K_{1}^{2}\right) & \text { for } r>r_{0}
\end{array},\right.
\end{gathered},
$$

and for the polarization in the $U-V$ plane we get

$$
\left(\begin{array}{l}
U \\
V
\end{array}\right)=P(\bar{r})\left(\begin{array}{c}
-y \\
x
\end{array}\right), \quad P=\frac{2 \operatorname{sgn} m_{i}}{r}\left\{\begin{array}{ll}
\left(a K_{0}+b I_{0}\right)\left(-a K_{1}+b I_{1}\right) & \text { for } r \leq r_{0} \\
c^{2} K_{0} K_{1} & \text { for } r>r_{0}
\end{array} .\right.
$$

This agrees for $r>r_{0}$ with the result for circular system without an edge in Eq. (13), since the Dirac mass is $-m_{i}$. Inside the edge for $r \leq r_{0}$ the situation is different, though. As visualized in Fig. 4, the vorticity changes from a counterclockwise vorticity to a clockwise vorticity as we get closer to the center of the circle, because the Dirac mass is $m_{i}>0$ and the factor $\left(a K_{0}+b I_{0}\right)\left(-a K_{1}+b I_{1}\right)$ becomes negative for small $\bar{r}$. Near the circular edge, however, there is a counterclockwise vorticity. Therefore, the vorticity near the edge behaves in analogy with the orientation of the polarization near a straight edge (cf. Eq. (17) and Fig. 2).

\section{Simply connected geometry with a circular edge}

Adding a concentric circular edge to the geometry of Fig. 1b results again in two regions which are separated by the edge (cf. Fig. 3b). In order to get a continuous spinor $\mathbf{E}$ from the solution (14), the mass sign of the second component must be compensated. Moreover, an extra factor must take care of the fact that the exponential function is continuous at the edge, which is possible by replacing

$$
e^{-\left|m_{i}\right| r} \rightarrow e^{-\left|m_{i}\right|\left|r-r_{0}\right|} \text {. }
$$

Thus, we obtain

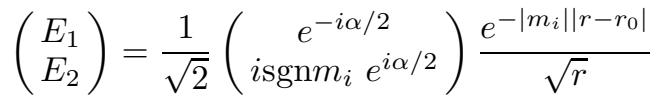




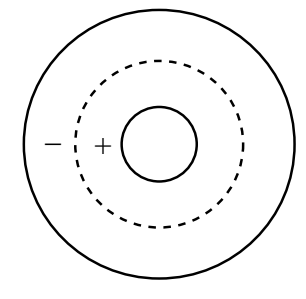

a

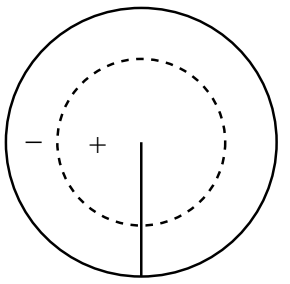

b

FIG. 3: Geometry of circular samples of Fig. 1 with a circular edge (dashed circle).

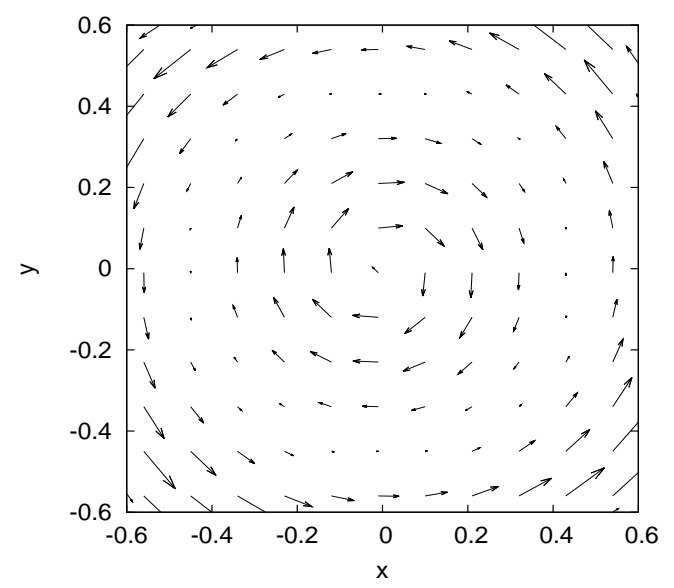

FIG. 4: The $U-V$ projection of the polarization field for positive $m_{i}$ inside the circular edge with radius $r_{0}=1$ for the geometry of Fig. 3a: the edge, together with the inner circular boundary, creates a double vortex. (Sect. IIIB). The polarization is normalized by the intensity here.

as the zero energy solution of the Dirac equation. Despite of the radial cut, the intensity has no angular dependence and reads

$$
I=\frac{e^{-2\left|m_{i}\right|\left|r-r_{0}\right|}}{r}
$$

As a typical example the radial intensity profile is plotted in Fig. 5 with a cusp at the edge. The intensity decays exponentially on the scale $1 /\left|m_{i}\right|$ away from the edge. On the other hand, at the center $r=0$ the intensity diverges like $1 / r$, although its spatial integral (i.e., the total intensity) is finite:

$$
\int_{0}^{\infty} \operatorname{Irdr}=\frac{2-e^{-2\left|m_{i}\right| r_{0}}}{\left|m_{i}\right|} .
$$

Moreover, $Q=0$ and the remaining two Stokes parameters read

$$
\left(\begin{array}{c}
U \\
V
\end{array}\right)=\operatorname{sgn} m_{i}\left(\begin{array}{c}
-y \\
x
\end{array}\right) \frac{e^{-2\left|m_{i}\right|\left|r-r_{0}\right|}}{r^{2}},
$$

representing a vortex whose vorticity changes with the sign of the Dirac mass. In contrast to the case with two boundaries of Sect. III B, the vorticity does not change its sign as a function of the radius. Moreover, the Stokes parameters are not affected by the radial cut, which is similar to the result for the Dirac eq. without an edge in Eq. (15). 


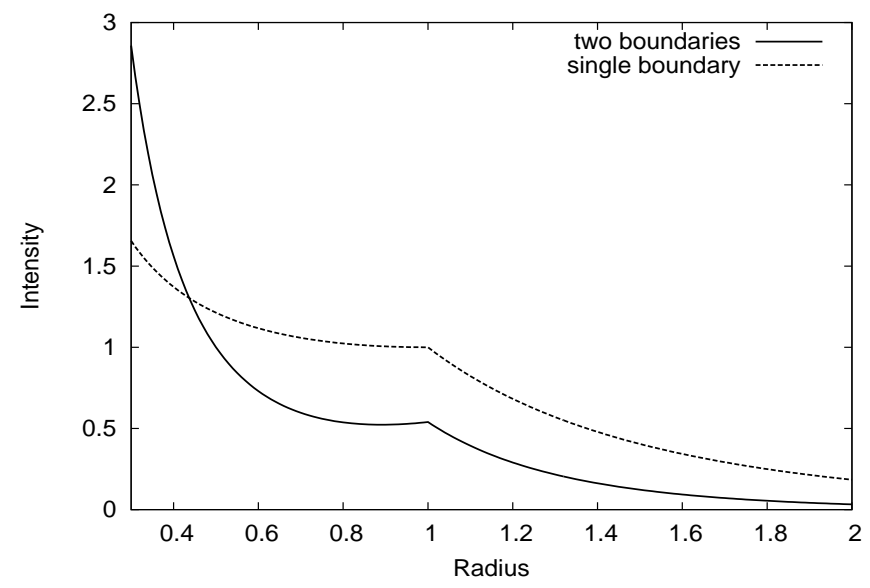

FIG. 5: The radial intensity profile has a cusp from the edge at $r_{0}=1$, according to the expressions (21) and (26).

\section{DISCUSSION}

The polarization of a 2D two-component electromagnetic field near a single gapped Dirac cone is analyzed under different boundary conditions, with and without a circular edge. Without an edge there are two characteristic solutions, depending on the sample geometry proposed in Fig. 1: For a sample with a hole there is a three-component polarization field $(U, V, Q)$ with a vortex in the $U-V$ plane. The solution for the geometry with a single boundary, on the other hand, is a two-component polarization vortex field inside the $U-V$ plane. The vorticity depends in both cases on the sign of the Dirac mass. This is important, since neither the Dirac spectrum, the intensity $|\mathbf{E}|^{2}$ nor the Stokes parameter $Q$ depend on the sign of the Dirac mass. Therefore, the polarization inside the $U-V$ plane plays a similar role as the Berry connection or the Chern number for the spinor field $\mathbf{E}$ as a characterization of the mass sign.

An edge in the form of a step-like sign change of the Dirac mass is usually accompanied by the formation of an edge state, which decays exponentially off away from the edge. This is also observed in the case of a circular geometry. In our examples there are several edge effects: The vortex is only affected by the edge in the case of the geometry with two boundaries, as visualized in Fig. 4, whereas for the geometry with a single boundary the vortex structure is the same with or without the edge. The reason for this difference is that in the former case there are three competing effective edges, namely the two boundaries and the edge of the changing mass. This leads to the formation of two different vorticities near the edge.

The structure of the polarization can also be characterized by the Berry curvature $[16,18]$

$$
B=\frac{1}{2} \mathbf{n} \cdot\left[\partial_{x} \mathbf{n} \times \partial_{y} \mathbf{n}\right],
$$

where $\mathbf{n}$ is the normalized polarization on the unit sphere of Eq. (6). $B$ vanishes for the simply connected geometry with and without an edge because of $Q=0$, meaning that the polarization is flat. On the other hand, $B$ is a non-vanishing function of the radius $r$ for the geometry with two boundaries. The Berry connection of the spinor field $\mathbf{E}$ is non-zero in both cases, though, and it corresponds with the projection of the polarization vortex onto the $U-V$ plane.

As mentioned in the Introduction, the results of these calculations are applicable to electronic systems with a Dirac node when the Coulomb interaction is neglected. Then the Stokes parameters $(U, V, Q)$ are local current densities defined in Eq. (5) with $\mathbf{E}$ replaced by the electronic spinor, since the components of the current operator are proportional to the Pauli matrices. Another interesting realization of circular edges are systems with polaritons [24]. 


\section{CONCLUSIONS}

Starting from the Dirac eq. (2) for a two-component electromagnetic field E, we have discussed solutions in the presence of two different types of boundaries (cf. Fig. 1) and with a circular edge caused by a mass sign change (cf. Fig. 3). From the electromagnetic field $\mathbf{E}$ the three-component polarization field was calculated with the result that a boundary creates a polarization field with a vortex, whose vorticity is proportional to the sign of the Dirac mass. In the presence of two boundaries and an edge the competition of regions with different signs of the Dirac mass creates a structure with changing vorticities (cf. Fig. 4). The Berry curvature of the polarization field depends on whether there is a sample geometry with one or two boundaries. This indicates that the polarization field carries substantial information which can be a used for measuring the properties of the edge states as well as for storing information by controlling the boundaries.

Acknowledgment: I am grateful to A. Genack for introducing me to his experiments on microwave metamaterials. This work was supported by a grant of the Julian Schwinger Foundation.

[1] K.S. Novoselov et al., Nature 438, 197 (2005).

[2] F.D.M. Haldane and S. Raghu, Phys. Rev. Lett. 100, 013904 (2008); S. Raghu and F.D.M. Haldane, Phys. Rev. A 78, 033834 (2008).

[3] X. Zhang, Phys. Rev. Lett. 100, 113903 (2008).

[4] T. Ochiai and M. Onoda, Phys. Rev. B 80, 155103 (2009)

[5] L.G. Wang, Z.G. Wang, J.X. Zhang and S.Y. Zhu, Opt. Lett. 34, 1510-1512 (2009).

[6] S.R. Zandbergen and M.J.A. de Dood, Phys. Rev. Lett. 104, 043903 (2010).

[7] X. Huang, Y. Lai, Z. H. Hang, H. Zheng and C.T. Chan, Nature Materials 10, 582-586 (2011)

[8] M.C. Rechtsman, J.M. Zeuner, Y. Plotnik, Y. Lumer, D. Podolsky, F. Dreisow, S. Nolte, M. Segev, and A. Szameit, Nature 496, 196 (2013)

[9] T. Ma, A.B. Khanikaev, S. Hossein Mousavi, and G. Shvets, PRL 114, 127401 (2015).

[10] P. Titum, N. Lindner, M.C. Rechtsman and G. Refael, Phys. Rev. Lett. 114, 056801 (2015).

[11] X. Cheng, C. Jouvaud, X. Ni, S. Hossein Mousavi, A.Z. Genack \& A.B. Khanikaev, Nature Mater. 15, 542-548 (2016).

[12] F.D.M. Haldane, Phys. Rev. Lett. 61, 2015 (1988).

[13] I. Campana, G. Jug and K. Ziegler, Phil. Mag., 86, 1667 (2006).

[14] A. Hill, A. Sinner and K. Ziegler, New Journ. Phys. 13, 035023 (2011).

[15] R. Courant and D. Hilbert, Methoden der Mathematischen Physik Vol. II (Springer Verlag).

[16] M.V. Berry, Proc. R. Soc. London Ser A 392, 45 (1984).

[17] R. de Gail, J.-N. Fuchs, M.-O. Goerbig, F. Piechon and G. Montambaux, Physica B Condensed Matter 407, 1948 (2012).

[18] F.D.M. Haldane, Optics Lett. 11, 730 (1986).

[19] S. John, Phys. Rev. Lett. 87, 2486 (1987).

[20] V.P. Bykov, Soviet Journal of Experimental and Theoretical Physics 35, 269 (1972); K. Ohtaka, Phys. Rev. B 19, 5057 (1979); E. Yablonovitch, Phys. Rev. Lett. 58, 2043 (1987).

[21] H.C. van de Hulst, Light Scattering by Small Particles, Dover Publications (New York 1981).

[22] M.I. Mishchenko, L.D. Travis and A.A. Lacis, Multiple scattering of light by particles (Cambridge University Press 2006).

[23] K. Ziegler, Journ. Phys. A: Math. and Theor. Phys., (2017).

[24] T. Karzig, C.-E. Bardyn, N. Lindner, G. Refael, Phys. Rev. X 5, 031001 (2015). 